\title{
A genome-wide transcriptional profiling of sporulating Bacillus subtilis strain lacking PrpE protein phosphatase
}

\author{
Adam Iwanicki $\cdot$ Krzysztof Hinc $\cdot$ Anna Ronowicz • \\ Arkadiusz Piotrowski • Aleksandra Wołoszyk • \\ Michał Obuchowski
}

Received: 26 February 2013/Accepted: 15 June 2013/Published online: 4 July 2013

(C) The Author(s) 2013. This article is published with open access at Springerlink.com

\begin{abstract}
The sporulation process is a complex genetic developmental program leading to profound changes in global gene expression profile. In this work, we have applied genome-wide microarray approach for transcriptional profiling of Bacillus subtilis strain lacking a gene coding for PrpE protein phosphatase. This protein was previously shown to be involved in the regulation of germination of B. subtilis spores. Moreover, the deletion of prpE gene resulted in changing the resistance properties of spores. Our results provide genome-wide insight into the influence of this protein phosphatase on the physiology of B. subtilis cells. Although the precise role of PrpE in shaping the observed phenotype of $\triangle p r p E$ mutant strain still remains beyond the understanding, our experiments brought observations of possible indirect implication of this protein in the regulation of cell motility and chemotaxis, as well as the development of competence. Surprisingly, prpE-deleted cells showed elevated level of general
\end{abstract}

Communicated by M. Hecker.

A. Iwanicki and K. Hinc contributed equally to this work.

Electronic supplementary material The online version of this article (doi:10.1007/s00438-013-0763-7) contains supplementary material, which is available to authorized users.

A. Iwanicki · K. Hinc · A. Wołoszyk · M. Obuchowski ( $\square)$ Laboratory of Molecular Bacteriology, Intercollegiate Faculty of Biotechnology, University of Gdańsk and Medical University of Gdańsk, Dębinki 1, 80-211 Gdańsk, Poland

e-mail: obuchowk@biotech.ug.gda.pl

A. Ronowicz · A. Piotrowski

Department of Biology and Pharmaceutical Botany,

Medical University of Gdańsk, Hallera 107,

80-416 Gdańsk, Poland stress response, which turned out to be growth medium specific.

Keywords Bacillus subtilis - Sporulation - PrpE protein phosphatase $\cdot$ Microarray $\cdot$ Transcriptional profiling

\section{Introduction}

Bacillus subtilis is able to form endospores in response to unfavorable environmental conditions. In the process of sporulation, bacterial cell undergoes asymmetric division resulting in the formation and subsequent release of highly durable spore. Sporulation is a complicated developmental process involving profound changes in gene expression profile. These changes are controlled by a cascade of $\sigma$ factors of RNA polymerase as well as various transcription factors. Main transcription factor responsible for entry into sporulation is Spo0A (Fawcett et al. 2000; Molle et al. 2003). The activity of this protein is regulated through phosphorylation by phosphorelay that integrates environmental and physiological signals in decision to sporulate (Jijang et al. 2000). In its phosphorylated state, Spo0A targets genes involved in the formation of a polar septum (Levin and Losick 1996) as well as genes driving activation of first two compartment-specific sigma factors of RNA polymerase, $\sigma^{\mathrm{E}}$ and $\sigma^{\mathrm{F}}$. The $\sigma^{\mathrm{F}}$ factor is activated only in the forespore compartment and $\sigma^{\mathrm{E}}$ in the mother cell. The activation of $\sigma^{\mathrm{F}}$ is critical for all subsequent spore development and is regulated by a complex mechanism (Hilbert and Piggot 2004). As the process of sporulation progresses these sigma factors become replaced by $\sigma^{\mathrm{G}}$ (forespore) and $\sigma^{\mathrm{K}}$ (mother cell) (Stragier and Losick 1996).

The mature spore is highly resistant to harsh environmental conditions (Nicholson et al. 2000; Takamatsu and 
Watabe 2002). It can remain dormant for very long periods of time and re-initiate vegetative growth in the process of germination once the environment becomes growth favorable. Spore can constantly monitor the environment and respond to the availability of nutrients or other germination factors (Moir et al. 2002; Setlow et al. 2003; Shah et al. 2008). Breaking of spore dormancy depends on different pathways depending on the conditions. In case of some germinants, for example L-alanine or an AGFK mixture (asparagine, glucose, fructose, potassium), spore germination depends on specific germination receptors encoded by three operons: gerA, gerB and gerK (Corfe et al. 1994a, b; Moir et al. 1994; Zuberi et al. 1987). In case of other germination inducing factors the receptors remain undiscovered. Expression of genes coding for germination receptors takes place only in the forespore compartment and reaches a maximum about 4-6 $\mathrm{h}$ upon initiation of sporulation (Corfe et al. 1994b; Feavers et al. 1990).

The PrpE protein phosphatase has been shown to influence the process of spore germination in B. subtilis. Spores of a strain deleted for the $\operatorname{prp} E$ gene are not able to initiate germination in response to $\mathrm{L}$-alanine. The expression of the gerA gene coding for germination receptor was originally shown to be significantly lowered in this strain. Moreover, spores of $\triangle p r p E$ strain are less resistant for desiccation as compared to the wild-type strain (Hinc et al. 2006). All these data point to the role of PrpE in regulation of spore formation as well as germination. It is worth adding, that the analysis of amino acid sequence of the PrpE protein shows the presence of motifs characteristic for protein phosphatases of PPP family as well as diadenosine polyphosphate hydrolases. Both activities of PrpE have been experimentally shown in vitro (Iwanicki et al. 2002).

Analysis of global changes in gene expression profiles has become a routine technique in molecular biology. This also accounts for microbiology, where DNA microarrays covering all annotated genes of various bacteria are commercially available. The progress of microarray technology is reflected in the number of works published involving the usage of this technique. B. subtilis has also been subjected to transcription profiling in various conditions such as sporulation (Fawcett et al. 2000), germination (Keijser et al. 2007), cold-shock response (Kaan et al. 2002) or phosphate limitation (Botella et al. 2011).

In this work, we have performed a transcriptional analysis of sporulating $\Delta p r p E$ strain. We used a DNA microarray technique to compare transcriptional profiles of wild-type 168 strain and $\triangle p r p E$ strain during sporulation. The results showed that over 490 genes were significantly upregulated in $\triangle p r p E$ strain, while another 220 genes showed downregulation. Most of upregulated genes belong to the general stress sigma factor regulon and $\sigma^{\mathrm{D}}$ regulon responsible for motility and chemotaxis. The group of significantly downregulated genes was not as homogenous but we have found that the part of SinR regulon encompassing genes responsible for the production of exopolysaccharide showed lower expression level in $\triangle p r p E$ strain. Moreover, we have found that the elevated level of the $\sigma^{\mathrm{B}}$ regulon expression is media dependent.

\section{Materials and methods}

\section{Bacterial strains}

Bacillus subtilis 168 (Anagnostopoulos and Crawford 1961) was used as a wild-type strain. Strain lacking PrpE phosphatase $(\triangle p r p E)$ was originally constructed in 168 genetic background (Iwanicki et al. 2002). A strain 2458 with genetic background of 168 harboring spoIIQ promoter fused to GFP encoding gene in non-essential locus amyE was a kind gift from prof. Ezio Ricca. Chromosomal DNA of this strain was used to transform $\triangle p r p E$ strain resulting in a strain BKH106 with following genotype $\Delta p r p E$ amyE:: $p_{\text {spoIIQ }}$-gfp $\mathrm{cm}^{\mathrm{r}}$. A strain PB198 with genetic background of 168 harboring ctc-lacZ fusion in amyE locus was obtained from prof. Chester Price (Boylan et al. 1993). A strain BAW01 was constructed by transformation of strain $\triangle p r p E$ with chromosomal DNA of PB198 resulting in $\Delta$ prpE amyE::ctc-lacZ $\mathrm{cm}^{\mathrm{r}}$ genotype.

\section{Sporulation conditions}

Sporulation was induced using the resuspension method (Harwood and Cutting 1990). Cells were grown overnight in Sterlini-Mandelstam growth medium. On the next day, cultures were refreshed in fresh growth medium at an $\mathrm{OD}_{600}=0.05$ and grown at $37^{\circ} \mathrm{C}$ until they reached an $\mathrm{OD}_{600}=0.6$. Cultures were centrifuged at $5,000 \times g$ for $5 \mathrm{~min}$ and resuspended in equal volumes of warm resuspension medium. The point of resuspension was defined as $T_{0}$.

RNA isolation, labeling, hybridization and scanning

RNA was isolated from sporulating bacteria of wild-type 168 and $\triangle p r p E$ strains collected at indicated time points. $10 \mathrm{OD}_{600}$ units of cultures were centrifuged at $5,000 \times g$ for $5 \mathrm{~min}$ and pellets were stored at $-80{ }^{\circ} \mathrm{C}$. Isolation of RNA was performed using Universal RNA Purification Kit (Eurx Ltd., Gdańsk, Poland) according to the manufacturer's protocol with following modification. Prior to extraction, bacterial pellet was resuspended in $300 \mu \mathrm{l}$ of TE buffer prepared on DEPC-treated water. $100 \mathrm{mg}$ of acid-washed glass beads (Sigma, USA) were added and the suspension was vortexed in Mini-BeadBeater (Biospect Products Inc., Bartlesville, OK, USA) ten times for $30 \mathrm{~s}$ with $1 \mathrm{~min}$ of 
incubations on ice between the pulses. Next, the mixture was centrifuged $12,000 \times g$ for $10 \mathrm{~min}$ and $100 \mu \mathrm{l}$ of supernatant was used for RNA extraction. DNA was removed by on-column DNase digestion with RNase-free DNase I (Thermo Fisher Scientific Inc., Waltham, MA, USA) as indicated in the manufacturer's protocol. The concentration and purity of the extracted RNA was assessed using NanoDrop ND-1000 spectrophotometer (Thermo Fisher Scientific) and the quality was checked by gel electrophoresis. $1 \mu \mathrm{g}$ of total RNA was used for the synthesis of double-stranded cDNA using Roche cDNA Synthesis System kit (Roche, Basel, Switzerland) using random hexamers (Thermo Fisher Scientific) as primers in the reverse transcription reaction. $250 \mathrm{ng}$ of double-stranded cDNA was labeled using Roche Dual-Color DNA Labeling kit (Roche), 168 strain-Cy5, $\Delta p r p E-$ Су3). Hybridization was performed using NimbleGen $4 \times 72 \mathrm{k} B$. subtilis subsp. subtilis strain 168 NC_000964 microarray slides following standard operating protocol (Roche NimbleGen Inc.). Each slide consisted of four separate hybridization fields covering whole transcriptome of $B$. subtilis with eight 60-nucleotide probes per gene in two technical replicates. Scanning was performed using Roche MS 200 Microarray Scanner following standard operating protocol (Roche). Three independent biological replicates have been performed for each strain.

\section{Data analysis}

The raw data (.pair file) were subjected to RMA (robust multi-array analysis) (Irizarry et al. 2003), including quantile normalization (Bolstad et al. 2003), and background correction as implemented in the NimbleScan software package, version 2.4.27 (Roche NimbleGen, Inc.). Normalized data were stored as Microsoft Excel spreadsheets. The SAM analysis (significance analysis of microarrays, Tusher et al. 2001) was performed with $\mathrm{MeV}$ package (Saeed et al. 2003) using data from three biological replicates adjusting delta parameter to keep the percentage of falsely positive genes below $1 \%$. The data were then averaged and used for further analyses. Fisher exact tests were performed with statistical $\mathrm{R}$ Package ( $\mathrm{R}$ Development Core Team 2011). The hierarchical clustering and $K$-means clustering was performed using $\mathrm{MeV}$ package. The raw and normalized data were deposited at GEO (NCBI) under accession GSE44125.

\section{$\beta$-Galactosidase measurements}

Cultures were grown at $37{ }^{\circ} \mathrm{C}$ with shaking. As a reach medium Difco nutrient broth (BD, Sparks, MD, USA) supplemented with $1 \mathrm{mM} \mathrm{Ca}\left(\mathrm{NO}_{3}\right)_{2}, 10 \mu \mathrm{M} \mathrm{MnCl}_{2}$ and $1 \mu \mathrm{M} \mathrm{FeSO}{ }_{4}$ was used. Samples were taken at indicated time points and stored at $-20{ }^{\circ} \mathrm{C}$ until enzyme assays were carried out. After thawing, bacterial pellets were suspended in buffer $\mathrm{Z}\left(60 \mathrm{mM} \mathrm{Na} 2 \mathrm{HPO}_{4}, 40 \mathrm{mM} \mathrm{NaH} \mathrm{PO}_{4}, 10 \mathrm{mM}\right.$ $\mathrm{KCl}, 1 \mathrm{mM} \mathrm{MgSO}_{4}$ ) containing $1 \mathrm{mM}$ dithiothreitol (DTT), and $1 / 100$ volume of lysis solution $(1 \mathrm{mg} / \mathrm{ml}$ DNase, $10 \mathrm{mg} / \mathrm{ml}$ lysozyme) was added. The mixture containing lysed cells was incubated for $20 \mathrm{~min}$ at $37^{\circ} \mathrm{C}$ and then centrifuged for $10 \mathrm{~min}$ at $10,000 \times g$ and $4{ }^{\circ} \mathrm{C}$ to remove the cell debris. The supernatant was used for measurement of protein concentration and $\beta$-galactosidase activity. Protein concentrations were measured using the Bradford reagent (Bio-Rad) as recommended by the manufacturer. Supernatants $(200 \mu \mathrm{l})$ were mixed with $600 \mu \mathrm{l}$ of buffer $\mathrm{Z}$ containing $1 \mathrm{mM}$ DTT. Samples were placed in a $37^{\circ} \mathrm{C}$ water bath, and $200 \mu \mathrm{l}$ of $o$-nitrophenyl- $\beta$-D-galactopyranoside $(4 \mathrm{mg} / \mathrm{ml})$ was added. The reaction was stopped by addition of $500 \mu \mathrm{l}$ of $1 \mathrm{M} \mathrm{Na}_{2} \mathrm{CO}_{3}$, and the absorbance was measured at $420 \mathrm{~nm}$. $\beta$-galactosidase activity, in nmol of $o$-nitrophenol produced $\min ^{-1} \mathrm{mg}^{-1}$, was calculated using the following formula: (absorbance at $420 \mathrm{~nm} \times 1.5) /($ concentration of protein in $\mathrm{mg} / \mathrm{ml} \times$ volume of sample in $\mathrm{ml} \times$ reaction time in $\min \times 0.00486$ ).

\section{Results}

Wild-type and $\triangle p r p E$ strains initiate sporulation process at the same time

Bacterial monocultures show a large degree of diversity by heterogeneous cell differentiation and population subculturing. Stochastic population splitting has been demonstrated for variety of stress responses including sporulation, competence or motility (Dubnau and Losick 2006; Smits et al. 2006). The heterogeneity in B. subtilis spore formation has been suggested to be a result of heterochronic phosphorelay gene expression (de Jong et al. 2010). We wanted to analyze whether deletion of the prpE gene influences timing of sporulation initiation. To check that the sporulation process in $\triangle p r p E$ strain initiates at the same time as in wild-type 168 strain, we have used a strain harboring $\sigma^{\mathrm{F}}$-dependent promoter of spoIIQ gene fused to gfp gene enabling for monitoring the progress of sporulation. The sporulation was induced as described in "Materials and methods" and the fluorescence of GFP protein was monitored. The results (Fig. 1) clearly indicate that both strains initiate sporulation at the same time.

Transcriptional profiling of sporulating $\triangle p r p E$ strain

Experiments were carried out with RNA from wild-type 168 cells and from cells mutant for PrpE. Cells were harvested 60, 130, 200, 270, 340 and 410 min upon initiation 


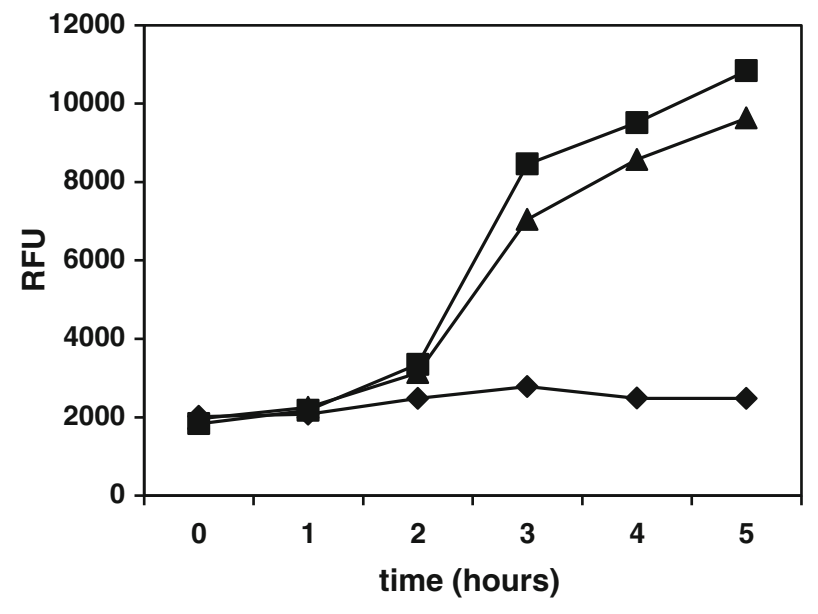

Fig. 1 The activity of $p_{\text {spolI }}$ promoter as assessed by measurement of GFP fluorescence. Bacteria were grown in Sterlini-Mandelstam medium and the fluorescence of GFP was measured at indicated times. Squares indicate strain 2458 (amyE:: $p_{\text {spoIIQ }}$ gfp), triangles strain BKH106 ( $\Delta p r p E$ amyE:: $p_{\text {spoIIQ }}$ gfp) and diamonds strain 168. Vertical axis values indicate relative fluorescence units, horizontal axis values time in hours upon induction of sporulation

of sporulation, which roughly corresponds to sporulation times $T_{1}, T_{2}, T_{3}, T_{4.5}, T_{5.5}$ and $T_{7}$. Isolated total RNA was reverse transcribed to cDNA and labeled. Next, labeled cDNAs from each time point were hybridized against DNA microarrays covering all annotated genes of B. subtilis. Scanned fluorescence values were processed as described in "Materials and methods". As a result, we obtained a panel of mutant to wild-type fluorescence ratios for each of time points. Upon the SAM analysis, we obtained 490 genes, which were significantly upregulated and 223 genes, which were significantly downregulated at least at one time point in $\Delta p r p E$ strain as compared to wild-type strain. These numbers represent 11.9 and $5.4 \%$ of all B. subtilis genes. The exact numbers of genes significantly up- and downregulated in $\triangle p r p E$ as compared to the wild-type strain at each time point tested are listed in Table 1 and the distributions of $\Delta p r p E /$ wild-type gene expression ratios are depicted as MA plots in Figure S1.

Functional analysis

Up- and downregulated genes were assigned their functional categories following classification proposed by Mäder et al. (2012). Based on this assignment, we assessed the overrepresentation of each functional category using Fisher exact test $(\alpha=0.05)$ applied on calculated gene expression ratios between $\triangle p r p E$ and wild-type strains. The individual functional categories were then subjected to hierarchical clustering using the technique of Eisen et al. (1998) (Fig. 2a, b). Following the same procedure genes were assigned to the regulons of transcription factors (Mäder et al. 2012) and the overrepresentation of each regulon was tested (Fig. 2c, d).

Expression of sporulation RNA polymerase $\sigma$ factors regulons

The prpE-deleted strain was shown to be unable to initiate germination of spores in response to some germination factors. Moreover, spores of $\triangle p r p E$ strain are significantly less resistant to dry heat treatment (Hinc et al. 2006). These observations prompted us to analyze the expression ratio patterns of genes belonging to regulons of sporulation $\sigma$ factors of RNA polymerase. Lists of genes under control of $\sigma^{\mathrm{F}}, \sigma^{\mathrm{E}}, \sigma^{\mathrm{G}}$ and $\sigma^{\mathrm{K}}$ were taken from Subtiwiki database (Mäder et al. 2012). The numbers of genes significantly up- and downregulated as assessed by SAM analysis listed in Table 2 indicated that the expression of only a minor percentage of these genes differs between the wild-type and $\Delta p r p E$ strains. This observation was more evident when average gene expression ratios of $\Delta p r p E /$ wild-type strain across all time points tested were represented as centroid graphs (Figure S2). We have additionally focused on the expression of gerA, gerB and gerK operons encoding germination receptors. A centroid graph of averaged $\Delta p r p E /$ wild-type expression ratios of genes forming each operon (Fig. 3) revealed that the level of expression of gerA operon does not differ between both strains. The expression of ger $B$ operon is elevated in $\Delta p r p E$ mutant as compared to the wild-type strain at 60 and 130-min time points and these differences are statistically significant. In case of gerK operon, although the expression in $\triangle p r p E$ strain seems to be lowered as compared to the wild-type strain, the difference is statistically significant only for $\operatorname{ger} K B$ and gerKC genes at 200-min time point.

Table 1 The numbers of genes with statistically significant difference in expression between $\triangle p r p E$ mutant and the wild-type strain as assessed by SAM analysis

\begin{tabular}{lcccccc}
\hline & $60 \min$ & $130 \mathrm{~min}$ & $200 \mathrm{~min}$ & $270 \min$ & $340 \mathrm{~min}$ & $410 \mathrm{~min}$ \\
\hline Upregulated & 145 & 88 & 490 & 160 & 44 & 206 \\
Downregulated & 18 & 6 & 223 & 89 & 19 & 51 \\
\hline
\end{tabular}


Expression of $\sigma^{\mathrm{D}}$ regulons in $\Delta p r p E$ mutant strain

Functional analysis revealed overrepresentation of upregulated genes of the category 4.1, Exponential and early post-exponential lifestyles, at most of time points in case of $\Delta p r p E$ mutant strain (Fig. 2a). Genes belonging to this category are controlled mainly by $\sigma^{\mathrm{D}}$ and are responsible for mobility and chemotaxis. Therefore, we observed the overrepresentation of upregulated genes of this sigma factor regulon in $\triangle p r p E$ strain as compared to the wild-type strain (Fig. 2c). The gene expression ratios for genes of $\sigma^{D}$ regulon represented in one graph along with gene expression data (Fig. 4) show that although transcription of this regulon in both strains follows the same pattern, its overall level in $\triangle p r p E$ strain is higher. The individual gene expression ratios of $\Delta p r p E /$ wild-type strains were subjected to $K$-means clustering. As a result we obtained ten groups of genes with similar expression ratio pattern (Fig. 5; Figure S3 and Table S1). The expression of genes in groups I ( $\operatorname{deg} R, f l g B, f l g C, f l E, y x k C, t l p C, y j c P, m c p C)$, II (fliG, mcpB, fliF, lytC, cheV, epr, flhP), and VIII (lytC, $f l g K, f g L, l y t B, y v y G, y v y F, f l g M, \operatorname{mot} A)$ is higher in $\triangle p r p E$
A

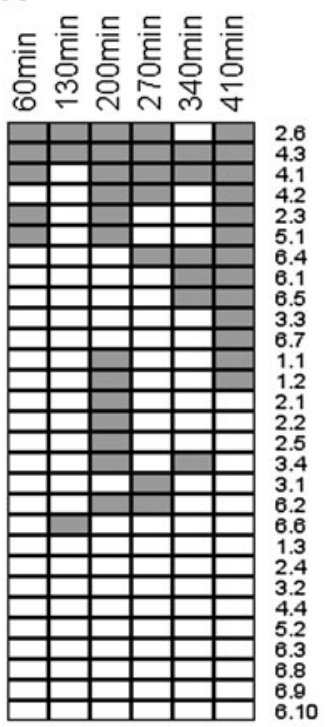

B

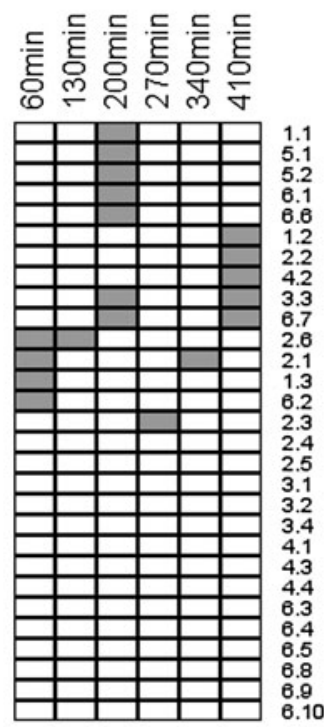

C
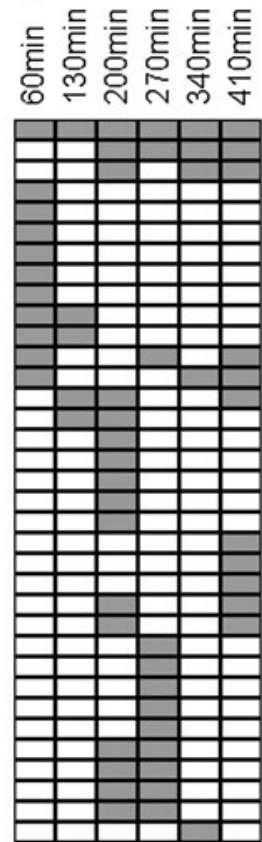

D
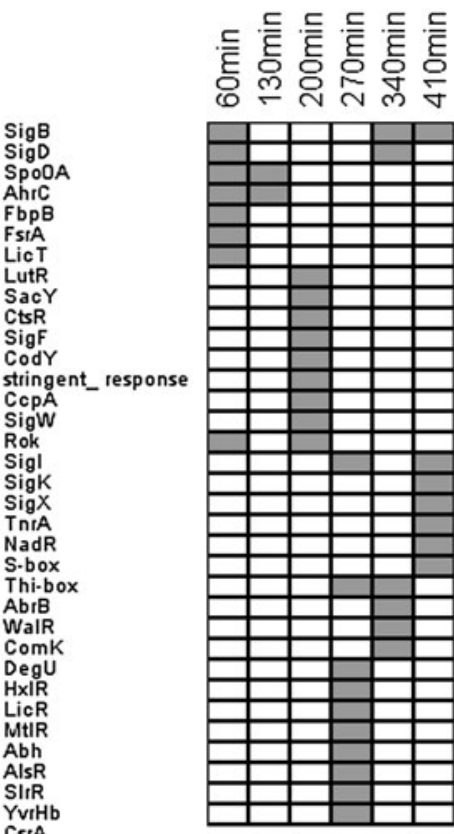

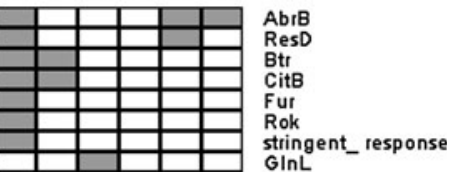

GInL

KipR

MhqR

OhrR
YclJ

Ydfl

Yess

YetL

L.box

AraR

GcpA

GmuR

SigE

DeoR

Abh

ArsR

SdpR

Ahrc

CatR

DnaA

EAR

GabR
RocR

SigL
Fig. 2 Hierarchical clustering of overrepresented functional categories of gene products (a, b) and regulons (c, d) (Mäder et al. 2012). a and c Upregulated genes, $\mathbf{b}$ and $\mathbf{d}$ downregulated genes. The categories are as follows: 1.1 cell wall and cell division, 1.2 transporters, 1.3 homeostasis, 2.1 electron transport and ATP synthesis, 2.2 carbon metabolism, 2.3 amino acid/ nitrogen metabolism, 2.4 lipid metabolism, 2.5 nucleotide metabolism, 2.6 additional metabolic pathways, 3.1 genetics, 3.2 RNA synthesis and degradation, 3.3 protein synthesis, modification and degradation, 3.4 regulation of gene expression, 4.1 exponential and early post-exponential lifestyles, 4.2 sporulation and germination, 4.3 coping with stress, 4.4 lifestyles/ miscellaneous, 5.1 prophages, 5.2 mobile genetic elements, 6.1 essential genes, 6.2 membrane proteins, 6.3 GTP-binding proteins, 6.4 phosphoproteins, 6.5 universally conserved proteins, 6.6 poorly characterized/putative enzymes, 6.7 proteins of unknown function, 6.8 short peptides, 6.9 ncRNA, 6.10 pseudogenes

Table 2 The numbers of genes with statistically significant difference in expression between $\triangle p r p E$ mutant and the wild-type strain belonging to following $\sigma$ factors regulons (Mäder et al. 2012) as assessed by SAM analysis

\begin{tabular}{llllllll}
\hline Regulon & $\begin{array}{l}\text { Number of genes } \\
\text { in regulon }\end{array}$ & $60 \mathrm{~min}$ & $130 \mathrm{~min}$ & $200 \mathrm{~min}$ & $270 \mathrm{~min}$ & $340 \mathrm{~min}$ \\
\hline$\sigma^{\mathrm{E}}$ & 171 & $5 / 0$ & $3 / 1$ & $16 / 8$ & $6 / 2$ & $2 / 2$ \\
$\sigma^{\mathrm{F}}$ & 62 & $7 / 0$ & $4 / 2$ & $5 / 2$ & $4 / 3$ & $1 / 1$ & $0 / 10$ \\
$\sigma^{\mathrm{G}}$ & 108 & $7 / 0$ & $6 / 0$ & $7 / 4$ & $3 / 1$ & $0 / 1$ \\
$\sigma^{\mathrm{K}}$ & 103 & $0 / 0$ & $2 / 0$ & $3 / 2$ & $0 / 2$ & $1 / 0$ & $1 / 1$ \\
$\sigma^{\mathrm{B}}$ & 159 & $54 / 0$ & $42 / 0$ & $84 / 5$ & $46 / 0$ & $13 / 0$ \\
$\sigma^{\mathrm{D}}$ & 81 & $0 / 0$ & $0 / 0$ & $23 / 0$ & $12 / 1$ & $6 / 0$ & $1 / 2$ \\
\end{tabular}

Numbers to the left of slash sign, upregulated genes; numbers to the right of slash sign, downregulated genes 


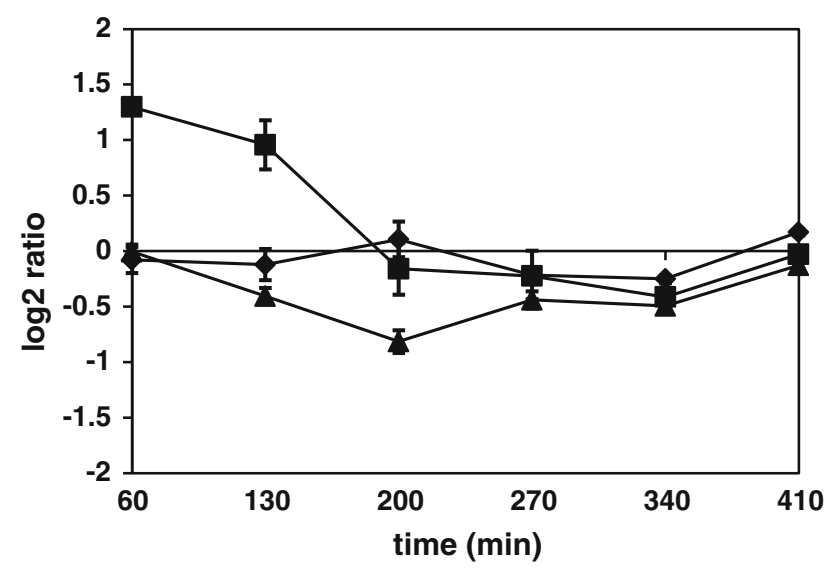

Fig. 3 Average $\Delta p r p E / 168$ gene expression ratios of germination receptors operons. Diamonds GerA operon, squares GerB operon, triangles GerK. Vertical axis values indicate normalized $\log _{2}$ of ratios, horizontal axis values time points upon induction of sporulation. Error bars indicate standard deviation of gene expression ratios

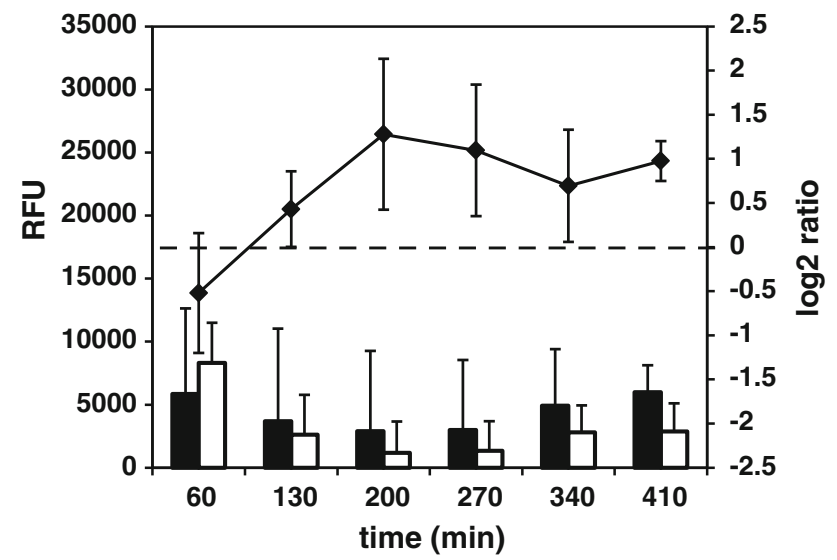

Fig. 4 Average gene expression and $\Delta p r p E / 168$ gene expression ratios of $\sigma^{\mathrm{D}}$ regulon. Closed columns gene expression in 168 wildtype strain, open columns gene expression in $\triangle p r p E$ mutant strain. Line graph $\Delta p r p E / 168$ gene expression ratios. Left vertical axis values gene expression shown as relative fluorescence units, right vertical axis values normalized $\log _{2}$ of ratios, horizontal axis values time points upon induction of sporulation. Error bars indicate standard deviation of gene expression or gene expression ratios

strain at all time points tested with the peak at 200-min time point. In case of groups III ( $f h F, c h e W, f h G$, cheA, cheD, sigD), IV (flgE, cheY, fliZ, fiP, dltB) and X (fliL, fliY, $f(i M)$ the expression of genes in mutant strain is more than twofold lower than in wild-type strain only at 60 -min time point and then turns to be higher across the rest of time points. Groups V (fliS, fliT, yfmT, yfmS, fliD), VI (hag, $y v y C, d l t C)$ and IX (fli, fliJ, fliK, ylxF, fliH, flqD) show only slight decrease of gene expression in $\triangle p r p E$ strain as compared to the wild type at 60 -min time point which then starts to be constantly higher. An individual gene expression ratio pattern is shown by genes of group VII ( $f h A$, che $B$, che $C$ ) which starts with about threefold decrease at 60-min time point in mutant strain, comparable expression at $130 \mathrm{~min}$ in both strains and slight increase in $\Delta p r p E$ strain at later time points.

Transcription of genes important for motility as well as extracellular matrix production is regulated by Spo0A. High levels of phosphorylated Spo0A repress flalche motility operon, while Spo0A-P is required for extracellular matrix gene expression via the activation of the regulatory protein SinI and, as a result, the transcriptional regulator SinR (Fujita et al. 2005). We have plotted $\Delta p r p E /$ wild-type gene expression ratios of $\sigma^{\mathrm{D}}$ and $\operatorname{SinR}$ regulons (Table S2) in one graph (Fig. 6). The averaged expression of SinR-dependent genes is decreased in $\triangle p r p E$ mutant as compared to the wild-type strain with the lowest level reached at 130-min time point. Since the level of Spo0A-P is controlled indirectly by a set of Rap phosphatases (Perego and Brannigan 2001; Pottathil and Lazazzera 2003) and directly by at least three other phosphatases: Spo0E (Perego and Hoch 1991; Ohlsen et al. 1994), YisI and YnzD (Perego 2001) it was interesting to compare the expression of genes coding these enzymes in wild-type and $\triangle p r p E$ strains. Results presented in Table 3 show $\triangle p r p E /$ wild-type strain expression ratios of these genes. While yisI and $y n z D$ are upregulated in $\Delta p r p E$ strain as compared to the wild type with statistically significant difference only at 200-min time point, the rapH gene is statistically significantly upregulated from 60 up to 270 min upon induction of sporulation.

Expression of genes involved in genetic competence in $\Delta p r p E$ mutant strain

Another functional category, which revealed upregulation of genes in $\triangle p r p E$ mutant strain in comparison to the wild type and is controlled by Spo0A (Grossman 1995), is the category 4.1, Lifestyles-genetic competence (Fig. 2a). The genes of this functional category show overall upregulation of expression in $\triangle p r p E$ mutant strain compared to the wild-type strain with the highest peak at 200-min time point (Fig. 7). Hierarchical clustering revealed that $\mathrm{co}$ $m E A$-comEC, comFA-comFC and comGA-comGG operons are in the cluster with similar expression ratio pattern (Figure S4 and Table S3). These operons are under control of ComK competence transcription factor. The expression of $\operatorname{comK}$ gene is also upregulated in $\triangle p r p E$ mutant cells compared to wild-type strain, with the highest peak at 270 min time point.

Elevated expression of $\sigma^{\mathrm{B}}$ regulon in $\triangle p r p E$ mutant strain is medium dependent

Following the functional analysis, we have found that the category 4.3, Coping with stress, is overrepresented at all 


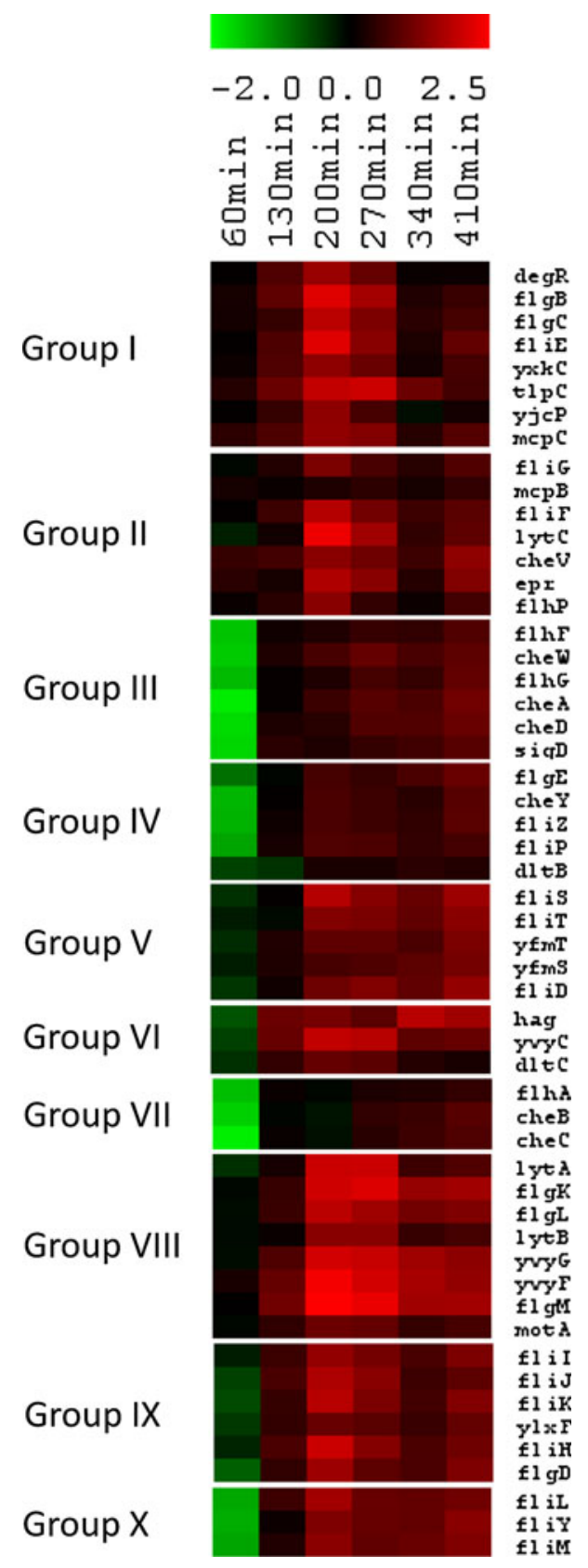

Fig. $5 K$-means clustering of $\Delta p r p E / 168$ gene expression ratios of $\sigma^{\mathrm{D}}$ regulon. Rows represent time points from 60 to $410 \mathrm{~min}$. Red and green indicate genes that are induced and repressed, respectively

time points tested (Fig. 2a). Consequently, the same observation was made for genes belonging to the regulon of the general stress response factor $\sigma^{\mathrm{B}}$ (Fig. 2c). The centroid graph of expression ratios of genes controlled by this sigma factor (159 genes) revealed overall upregulation of genes in $\triangle p r p E$ mutant compared to the wild-type strain at all of the time points but 410 min (Fig. 8). To further analyze the expression of genes of this regulon, we performed $K$-means clustering obtaining ten groups with

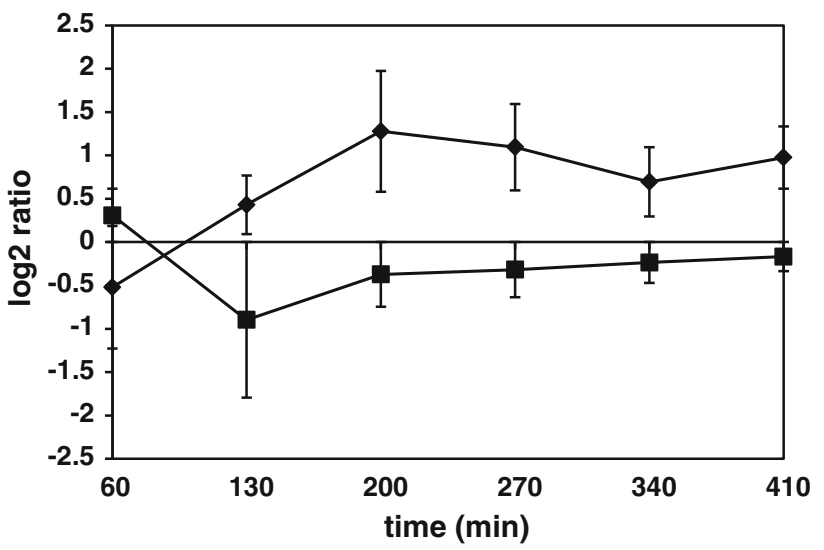

Fig. 6 Average $\Delta p r p E / 168$ gene expression ratios of $\sigma^{\mathrm{D}}$ and SinR regulons. Squares $\sigma^{\mathrm{D}}$ regulon, diamonds $\mathrm{SinR}$ regulon. Vertical axis values indicate normalized $\log _{2}$ of ratios, horizontal axis values time points upon induction of sporulation. Error bars indicate standard deviation of gene expression ratios

similar expression ratio patterns (Fig. 9; Figure S5 and Table S4). The group II ( $r n r, y i t T, y d a E$, trxA, yqhQ, ytkL, $k a t X, v h c M$ ) showed increased gene expression in $\triangle p r p E$ mutant compared to the wild-type strain only at 60 and 130-min time points. At later time points gene expression was comparable in both strains. The expression of genes in the group III ( $d p s, y c e E, y j b C, s p x, y c e D$, sodA, yvyD) was comparable in both strains at 60 -min time point, then it started to be elevated in the mutant strain with the peak at 200-min time point to return to close to the similar level at 410-min time point. The remaining groups show the same gene expression ratio patterns of upregulation at all the time points apart from the last one, $410 \mathrm{~min}$. The $\operatorname{sig} B$ gene by itself also shows clear upregulation in $\triangle p r p E$ mutant strain with the similar pattern.

We wanted to verify whether observed upregulation of $\sigma^{\mathrm{B}}$ regulon in $\triangle p r p E$ mutant strain was specific for sporulation process only. To do that we used the bacteria of both the wild-type and $\triangle p r p E$ strains harboring the promoter of known $\sigma^{\mathrm{B}}$-dependent gene $c t c$ fused transcriptionally with the reporter gene lac $Z$ in non-essential locus $a m y E$. These bacteria were grown in rich medium and the media used for the induction of sporulation and the activity of $\sigma^{\mathrm{B}}$-dependent promoter was assessed by $\beta$-galactosidase activity assay. As indicated in the Fig. 10a, the activity of ctc promoter follows the same pattern in both strains grown in reach medium with constant increase as the cultures are getting closer to the end of exponential growth phase and drop upon crossing the transition point. In case of media used for the induction of sporulation (Fig. 10b), the activity of $c t c$ promoter follows similar pattern; although in $\triangle p r p E$ mutant strain it is consequently higher across all time points tested. 
Table 3 Average $\log _{2} \Delta p r p E / 168$ gene expression ratios of genes encoding Spo0A-P phosphatases and Rap phosphatases

\begin{tabular}{|c|c|c|c|c|c|c|}
\hline Gene & $60 \mathrm{~min}$ & $130 \mathrm{~min}$ & $200 \mathrm{~min}$ & $270 \mathrm{~min}$ & $340 \mathrm{~min}$ & $410 \min$ \\
\hline spoOE & 0.36 & 0.62 & 0.54 & 0.59 & 0.29 & 0.14 \\
\hline$y i s I$ & 0.95 & 1.33 & $1.22 *$ & 0.81 & 0.28 & 0.25 \\
\hline$y n z D$ & 0.08 & 0.19 & $0.40 *$ & 0.24 & 0.14 & 0.03 \\
\hline rapA & -0.11 & 0.27 & 0.27 & 0.23 & 0.73 & $0.42 *$ \\
\hline$r a p B$ & 0.29 & 0.11 & 0.31 & 0.05 & -0.11 & -0.05 \\
\hline $\operatorname{rap} C$ & 0.31 & -0.31 & 0.15 & 0.14 & 0.18 & 0.64 \\
\hline rapD & -0.06 & -0.15 & -0.27 & -0.06 & 0.00 & -0.23 \\
\hline rapE & 1.04 & 0.47 & 0.43 & 0.39 & 0.03 & 0.25 \\
\hline rapF & -0.26 & -0.40 & 0.50 & 0.44 & 0.53 & 0.30 \\
\hline $\operatorname{rap} G$ & 0.01 & -0.31 & 0.36 & -0.17 & -0.28 & 0.10 \\
\hline rapH & $0.74 *$ & $0.38 *$ & $1.86^{*}$ & $1.61 *$ & 1.42 & 1.02 \\
\hline rapI & 0.29 & 0.38 & 0.81 & 0.50 & 0.06 & 0.04 \\
\hline rapJ & 0.32 & 0.34 & -0.40 & -0.26 & -0.06 & -0.09 \\
\hline rapK & 0.27 & 0.47 & $1.53^{*}$ & 1.19 & 0.25 & 0.26 \\
\hline
\end{tabular}

The numbers marked with asterisk results statistically significant as assessed by SAM analysis

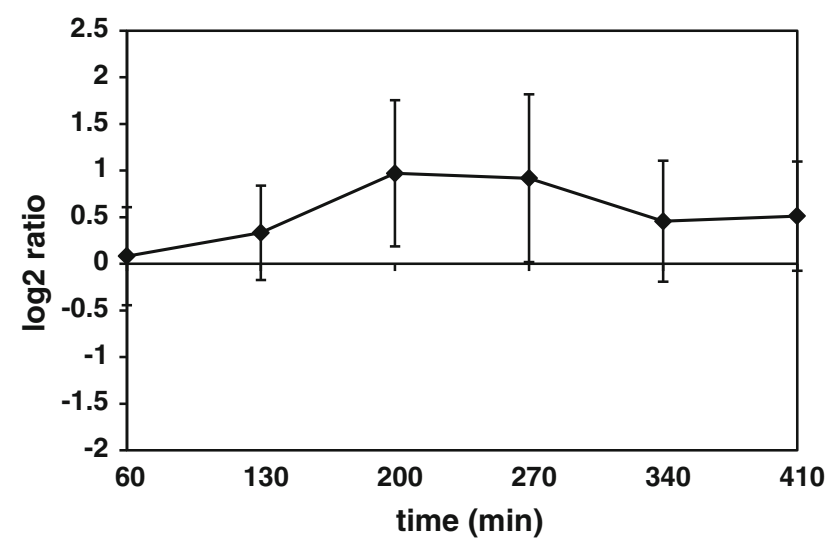

Fig. 7 Average $\Delta p r p E / 168$ gene expression ratios of genes involved in the development of the genetic competence. Vertical axis values indicate normalized $\log _{2}$ of ratios, horizontal axis values time points upon induction of sporulation. Error bars indicate standard deviation of gene expression ratios

\section{Discussion}

The process of spore formation is controlled by a multistep genetic program involving activity of four different sigma factors of RNA polymerase as well as other transcription factors. The microarray technique has widely been used to monitor global gene expression during this developmental process (e.g. Fawcett et al. 2000; Ohashi et al. 2003; Schmalisch et al. 2010; Steil et al. 2005). In spite of obvious advantages of this technique, it is important to be aware that heterogeneity of $B$. subtilis sporulation process can be a source of a bias or even incorrect conclusions drawn from the experiments conducted with improperly synchronized bacterial cultures. In our experiments, we

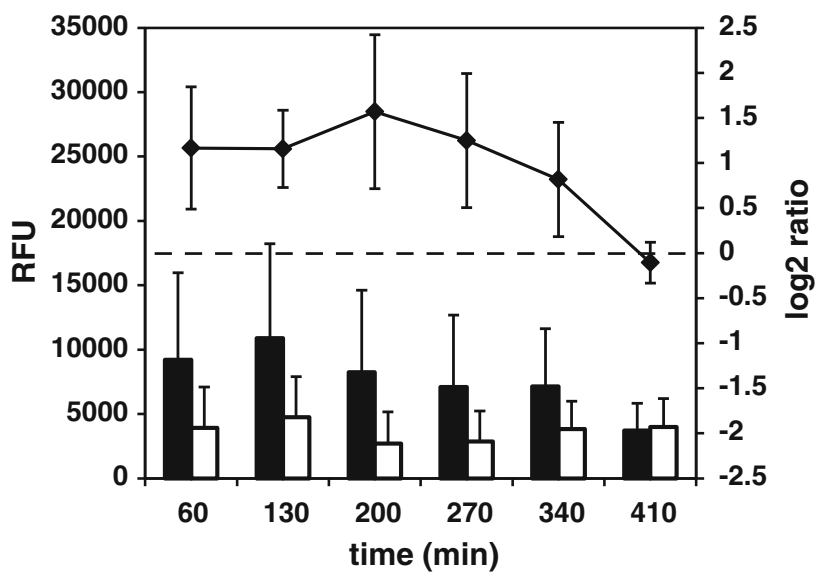

Fig. 8 Average gene expression and $\triangle p r p E / 168$ gene expression ratios of $\sigma^{\mathrm{B}}$ regulon. Closed columns gene expression in 168 wildtype strain, open columns gene expression in $\triangle p r p E$ mutant strain. Line graph $\Delta p r p E / 168$ gene expression ratios. Left vertical axis values gene expression shown as relative fluorescence units, right vertical axis values normalized $\log _{2}$ of ratios, horizontal axis values time points upon induction of sporulation. Error bars indicate standard deviation of gene expression or gene expression ratios

have used a method of inducing the sporulation widely approved as producing cultures of B. subtilis bacteria synchronically starting spore formation process. In addition, we have verified that both, wild-type and $\Delta p r p E$ mutant strains initiate sporulation at the same time and with the same efficiency (Fig. 1). The spoIIQ gene, which promoter we have used to express GFP protein in analyzed strains, is controlled by $\sigma^{\mathrm{F}}$ subunit and its product is required for spore engulfment (Londoño-Vallejo et al. 1997) and thus it seems to be suitable for monitoring the proceeding of the sporulation process. 
Fig. $9 K$-means clustering of $\Delta p r p E / 168$ gene expression ratios of $\sigma^{\mathrm{B}}$ regulon. Rows represent time points from 60 to $410 \mathrm{~min}$. Red and green indicate genes that are induced and repressed, respectively

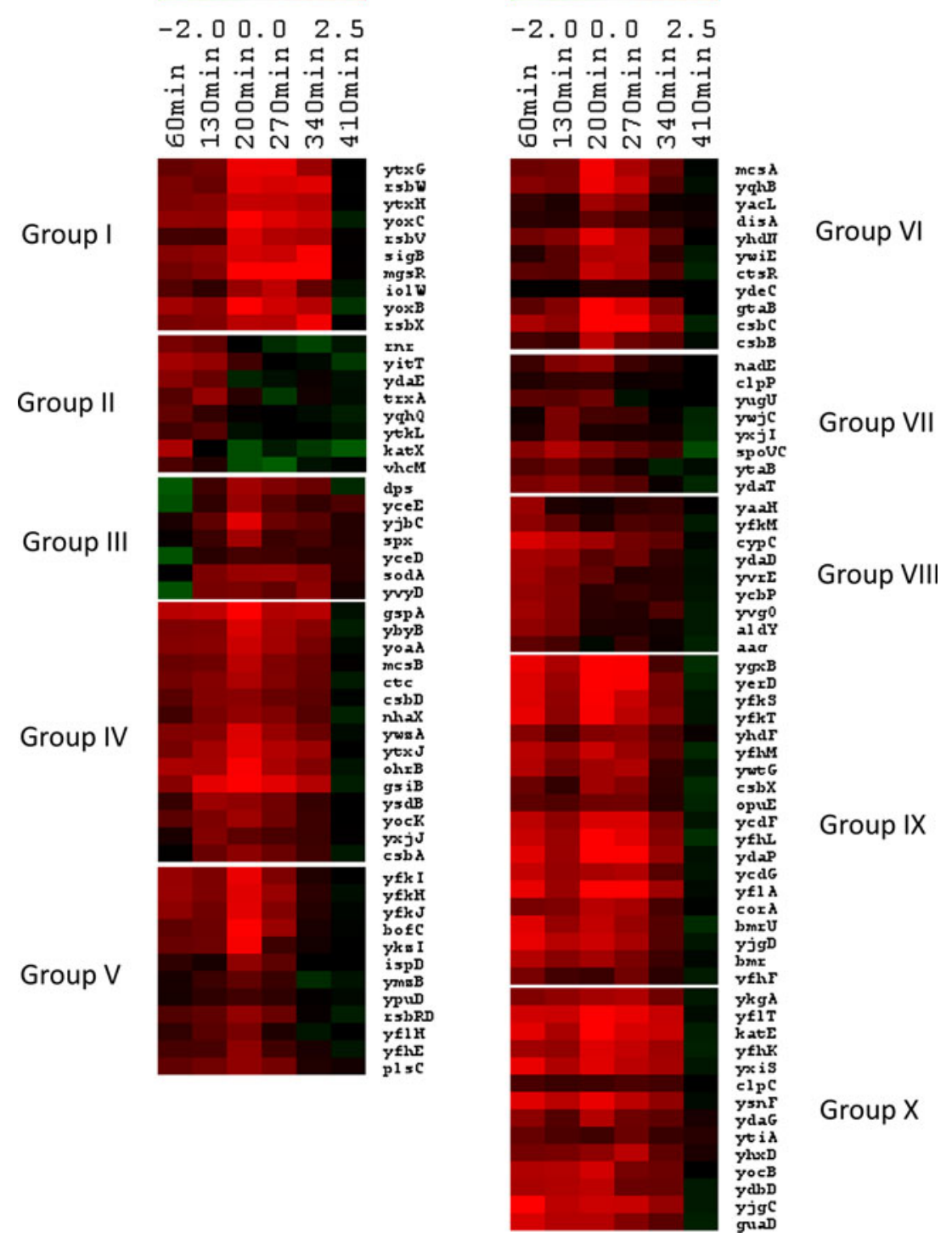

The PrpE protein phosphatase is thought to localize to the forespore compartment (Hinc et al. 2006), hence the deletion of the gene encoding this protein could potentially lead to altered expression of genes within the forespore. Although this hypothesis seems to be attractive, the results of transcriptional profiling of $\triangle p r p E$ mutant strain indicate that the expression levels of genes belonging to regulons of sporulation $\sigma$ factors are similar to the wild-type strain (Figure $\mathrm{S} 1$ ). Due to the fact that $\Delta p r p E$-produced spores are unable to initiate germination in response to L-alanine (Hinc et al. 2006), we additionally focused on the expression of germination receptors genes. The GerA receptor is thought to be responsible for induction of germination in response to L-alanine (Cabrera-Martinez et al. 2003), while GerB and GerK receptors were shown to respond to stimulation with a mixture of asparagine, glucose, fructose and potassium ions (Paidhungat and Setlow 2000). The expression of ger $A A$, ger $A B$ and ger $A C$ genes is unchanged in $\triangle p r p E$ mutant strain, while ger $B A$, ger $B B$ and ger $B C$ are statistically significantly upregulated in the mutant strain only at two earliest time points. These time points are very early for $\sigma^{\mathrm{G}}$-dependent genes, as $\sigma^{\mathrm{G}}$ regulon is expressed no earlier than $150 \mathrm{~min}$ upon induction of sporulation (Steil et al. 2005). Although the expression of GerK encoding genes seems to be lowered in $\Delta p r p E$ mutant as compared to the wild-type strain, observed differences are not statistically significant for whole operon and should not be taken into account. This observation partially contradicts our previously published data indicating that the expression of GerA receptor operon is lower in the $\Delta p r p E$ strain as compared to the wild-type strain (Hinc et al. 2006). This discrepancy most probably results from different methodology used, because in the previous work the expression of GerA operon was analyzed using indirect 


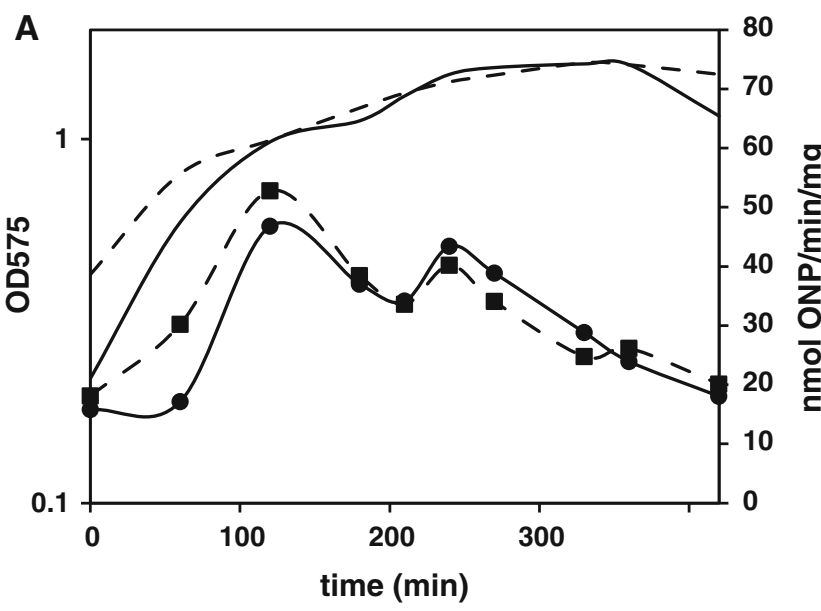

Fig. 10 Activity of $c t c$ gene promoter as assessed by $\beta$-galactosidase assay. a Rich medium, horizontal axis values indicate time upon refreshing overnight cultures. b Sterlini-Mandelstam and sporulation media, horizontal axis values indicate time upon refreshing overnight cultures relative to the resuspension of bacteria in sporulation medium indicated as 0 min. Solid line growth curve of strain PB198 (168 amyE::ctc-lacZ), dashed line growth curve of strain BAW01 ( $\Delta p r p E$

method of $\beta$-galactosidase activity measurement performed with a strain harboring $l a c Z$ reporter gene inserted into the gerAB-gerAC region. Moreover, according to recent literature data, it is suggested that the expression level of germination receptors determines the average rate but not the heterogeneity of spore germination (Zhang et al. 2013). Hence, no germination of $\triangle p r p E$ mutant spores in response to L-alanine does not have to correlate with lowered expression of appropriate germination receptor/receptors.

An interesting observation has been made for genes belonging to the regulon of $\sigma^{\mathrm{D}}$. The expression of $\sigma^{\mathrm{D}}$ dependent genes follows the same pattern in both $\Delta p r p E$ and wild-type strains, but its level in $\triangle p r p E$ mutant is elevated in comparison to the wild-type strain at $200 \mathrm{~min}$ and following time points (Fig. 4). The $\sigma^{\mathrm{D}}$ factor of $B$. subtilis is responsible for transcription of genes involved in motility and chemotaxis (Helmann et al. 1988; Márquez et al. 1990). It has been known for a long time that motility of sporulating $B$. subtilis decreases significantly (Nishihara and Freese 1975) and is reflected in rapid drop of $\sigma^{\mathrm{D}}$ activity upon initiation of sporulation process (Mirel and Chamberlin 1989). On the other hand, it is also known that motility, chemotaxis, development of competence and DNA uptake are alternative survival strategies of $B$. subtilis subjected to stress (Hamoen et al. 2003; Stragier 2006; Veening et al. 2008). The different cell fates are regulated by signaling network that relies primarily on the activity of three major transcriptional regulators: Spo0A, DegU and ComK (for review see Lopez et al. 2008).

A high level of DegU phosphorylation is required for the induction of exoprotease expression. In contrast, high

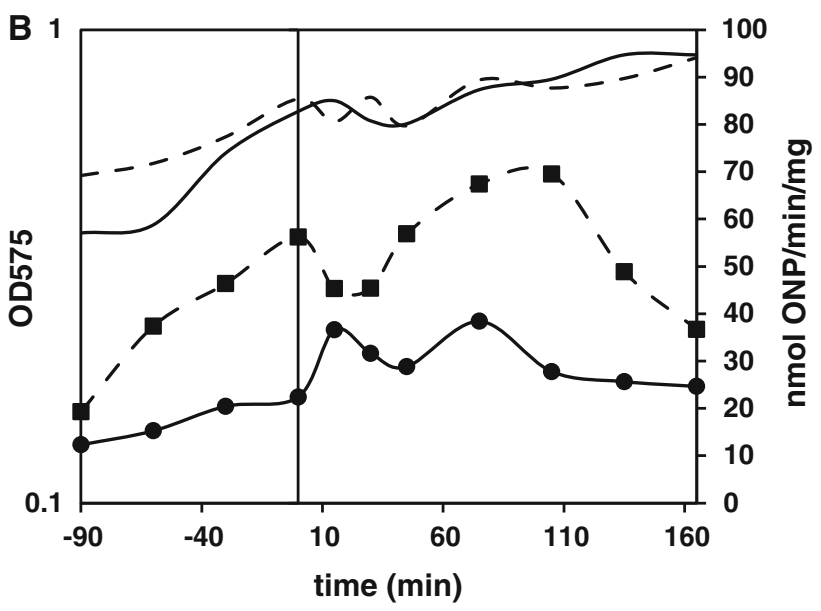

amyE::ctc-lacZ), solid line with circles $\beta$-galactosidase activity in strain PB198, dashed line with squares $\beta$-galactosidase activity in strain BAW01. Left vertical axis values indicate optical density of bacterial cultures measured at $575 \mathrm{~nm}$, right vertical axis value $\beta$ galactosidase activity expressed in nanomoles of $o$-nitrophenol produced per minute per $\mathrm{mg}$ of total protein. The results are the representatives of three independent experiments

levels of DegU-P repress motility as this protein directly binds the regulatory region of the fla/che motility and chemotaxis operon (Tsukahara and Ogura 2008). In our experiments, we have not observed differences in the expression of genes encoding extracellular degradative enzymes between $\triangle p r p E$ and the wild-type strains (data not shown). This leads to the conclusion that DegU probably does not directly contribute to elevated expression of $\sigma^{\mathrm{D}}$ regulon in prpE-deleted strain.

In the sessile state $B$. subtilis cells, especially those that form biofilms are able to produce extracellular matrix. Its production requires activation of two operons: epsA-O, responsible for production of the exopolysaccharide component and the yqxM-sipW-tasA operon, responsible for the production and secretion of the major protein component of the matrix (Branda et al. 2004; Kearns et al. 2005). These operons are controlled by an inhibitor, SinR. The activity of $\operatorname{SinR}$ is negatively regulated by a regulatory protein SinI, which is expressed only in cells in which Spo0A has been activated (Chai et al. 2008). On the other hand, high levels of Spo0A-P repress the fla/che operon (Fujita et al. 2005). Thus, the level of phosphorylated form of Spo0A in the cell can lead to a switch between motility and extracellular matrix production. The expression of SinR regulon is lowered in $\triangle p r p E$ mutant as compared to the wild-type strain (Fig. 6), which can correlate with elevated expression of $\sigma^{\mathrm{D}}$ regulon.

The elevated expression in $\triangle p r p E$ mutant as compared to the wild-type strain was also observed for genes involved in genetic competence (Fig. 7). The genetic competence development is governed by the master 
regulator ComK, which controls the initiation of the cascade of the genes involved in this process (Dubnau 1991; van Sinderen et al. 1995). The activation and accumulation of ComK is controlled by products of several genes including $a b r B$, $\sin R$ and $\operatorname{deg} U$ (Hahn et al. 1994; van Sinderen and Venema 1994). The control via AbrB is influenced by Spo0A, since the transcription of $a b r B$ is repressed by Spo0A-P $(78,104)$. According to the work of Mirouze and co-workers, Spo0A-P directly binds to the promoter region of $\operatorname{comK}$ and activates or represses its activity dependently on the concentration in the cell (Mirouze et al. 2012). Therefore, the upregulation of genes involved in development of genetic competence, especially ComK-dependent genes (Figure S3) observed in $\Delta p r p E$ strain may be explained by the regulatory action of Spo0A.

The results of our analysis point to the activity of Spo0A regulator as a probable cause of changed expression of above described groups of genes. The efficiency of spore formation as well as the timing of sporulation process seems to be unaffected in $\triangle p r p E$ strain (Hinc et al. 2006 and Fig. 1), hence any potential changes in the level of Spo0A-P in mutant cells should be rather discrete and most probably occur upon successful initiation of sporulation. The expression of $\operatorname{spoOA}$ gene is similar in both the strains $\triangle p r p E$ mutant and the wild type (data not shown). Our attention was drawn by phosphatases of Spo0A-P, which are responsible for inactivation of this protein. The genes encoding two direct phosphatases of Spo0A-P, spoOE and $y i s I$, and one gene coding for Rap phosphatase, rapH, involved in regulation of phosphorelay (Parashar et al. 2011) are upregulated in $\Delta p r p E$ strain. The combined action of these proteins may lead to more rapid drop of Spo0A-P level in $\triangle p r p E$ strain resulting in partial derepression of $\sigma^{\mathrm{D}}$ regulon and the genes involved in the development of genetic competence. As another effect of this drop, the repression of SinR regulon might occur via activation of SinR antagonist, SinI.

An unexpected observation has been made for genes belonging to the regulon of $\sigma^{\mathrm{B}}$, the key regulator of the general stress response in B. subtilis. Most of $\sigma^{\mathrm{B}}$-dependent genes are upregulated in $\Delta p r p E$ mutant strain (Fig. 8), which clearly suggests higher level of stress response in sporulating mutant bacteria. One could suggest that the shift of growing cultures from rich growth medium to very minimal sporulation medium, which takes place at the induction of sporulation procedure, may lead to a starvation and thus induction of stress response. We have verified this assumption by analyzing the expression of $c t c$, a wellknown general stress response genes, in bacteria growing in rich medium or media used for induction of sporulation. While the activity of $c t c$ promoter in rich medium did not differ in both strains (Fig. 10a), in Sterlini-Mandelstam (minimal) medium used for growing bacteria as well as very minimal resuspension medium used for induction of sporulation, the activity of $p_{c t c}$ was elevated in $\Delta p r p E$ mutant strain in the samples from each time point analyzed (Fig. 10b). This observation leads to the conclusions that elevated level of general stress response in the mutant strain is not sporulation specific. Moreover, it is connected with the type of media used for cultivation of bacteria. It seems plausible that the deletion of prpE gene leads to impaired utilization of amino acids as carbon source, nevertheless further investigation is needed for the verification of such hypothesis.

Taken together our results point to PrpE phosphatase as a protein involved in the regulation of processes described above via yet undefined mechanism, most probably influencing the activity of Spo0A regulator in sporulating $B$. subtilis cells. This hypothesis is an addition to former observations that the presence of this phosphatase seems to be important for establishing proper resistance of spores as well as for germination. Such conclusions place PrpE protein in the center of interest for research conducted on basic cellular and developmental processes of $B$. subtilis.

Acknowledgments This work was performed with financial support of Polish National Science Center, grant no. N301 152838.

Open Access This article is distributed under the terms of the Creative Commons Attribution License which permits any use, distribution, and reproduction in any medium, provided the original author(s) and the source are credited.

\section{References}

Anagnostopoulos C, Crawford IP (1961) Transformation studies on the linkage of markers in the tryptophan pathway in Bacillus subtilis. Proc Natl Acad Sci USA 47:378-390

Bolstad BM, Irizarry RA, Astrand M, Speed TP (2003) A comparison of normalization methods for high density oligonucleotide array data based on variance and bias. Bioinformatics 19:185-193

Botella E, Hübner S, Hokamp K, Hansen A, Bisicchia P, Noone D, Powell L, Saltzberg LI, Devine KM (2011) Cell envelope gene expression in phosphate-limited Bacillus subtilis cells. Microbiology 157:2470-2484

Boylan SA, Rutherford A, Thomas SM, Price CW (1993) Activation of Bacillus subtilis transcription factor sigma B by a regulatory pathway responsive to stationary-phase signals. J Bacteriol 174: 3695-3706

Branda SS, González-Pastor JE, Dervyn E, Ehrlich SD, Losick R, Kolter R (2004) Genes involved in formation of structured multicellular communities by Bacillus subtilis. J Bacteriol 186: 3970-3979

Cabrera-Martinez RM, Tovar-Rojo F, Vepachedu VR, Setlow P (2003) Effects of overexpression of nutrient receptors on germination of spores of Bacillus subtilis. J Bacteriol 185: 2457-2464

Chai Y, Chu F, Kolter R, Losick R (2008) Bistability and biofilm formation in Bacillus subtilis. Mol Microbiol 67:254-263

Corfe BM, Sammons RL, Smith DA, Mauel C (1994a) The gerB region of the Bacillus subtilis 168 chromosome encodes a 
homologue of the gerA spore germination operon. Microbiology 140:471-478

Corfe BM, Moir A, Popham D, Setlow P (1994b) Analysis of the expression and regulation of the ger $B$ spore germination operon in Bacillus subtilis. Microbiology 140:3079-3083

de Jong IG, Veening J-W, Kuipers OP (2010) Heterochronic phosphorelay gene expression as a source of heterogeneity in Bacillus subtilis spore formation. J Bacteriol 192:2053-2067

Dubnau D (1991) Genetic competence in Bacillus subtilis. Microbiol Rev 55:395-424

Dubnau D, Losick R (2006) Bistability in bacteria. Mol Microbiol 61:564-572

Eisen MB, Spellman PT, Brown PO, Botstein D (1998) Cluster analysis and display of genome-wide expression patterns. Proc Natl Acad Sci USA 95:14863-14868

Fawcett P, Eichenberger P, Losick R, Youngman P (2000) The transcriptional profile of early to middle sporulation in Bacillus subtilis. Proc Natl Acad Sci USA 97:8063-8068

Feavers IM, Foulkes J, Setlow B, Sun D, Nicholson W, Setlow P, Moir A (1990) The regulation of transcription of the gerA spore germination operon of Bacillus subtilis. Mol Microbiol 4:275-282

Fujita M, González-Pastor JE, Losick R (2005) High- and lowthreshold genes in the Spo0A regulon of Bacillus subtilis. J Bacteriol 187:1357-1368

Grossman AD (1995) Genetic networks controlling the initiation of sporulation and the development of genetic competence in Bacillus subtilis. Annu Rev Genet 29:477-508

Hahn J, Kong L, Dubnau D (1994) The regulation of competence transcription factor synthesis constitutes a critical control point in the regulation of competence in Bacillus subtilis. J Bacteriol 176:5753-5761

Hamoen LW, Venema G, Kuipers OP (2003) Controlling competence in Bacillus subtilis: shared use of regulators. Microbiology 149 :9-17

Harwood CR, Cutting SM (1990) Molecular biological methods for Bacillus. Wiley, New York

Helmann JD, Márquez LM, Chamberlin MJ (1988) Cloning, sequencing, and disruption of the Bacillus subtilis sigma 28 gene. J Bacteriol 170:1568-1574

Hilbert DW, Piggot PJ (2004) Compartmentalization of gene expression during Bacillus subtilis spore formation. Microbiol Mol Biol Rev 68:234-262

Hinc K, Nagórska K, Iwanicki A, Węgrzyn G, Séror SJ, Obuchowski M (2006) Expression of genes coding for GerA and GerK germination receptors is dependent on the protein phosphatase PrpE. J Bacteriol 188:4373-4383

Irizarry RA, Hobbs B, Collin F, Beazer-Barclay YD, Antonellis KJ, Scherf U, Speed TP (2003) Exploration, normalization, and summaries of high density oligonucleotide array probe level data. Biostatistics 4:249-264

Iwanicki A, Herman-Antosiewicz A, Pierechod M, Séror SJ, Obuchowski M (2002) PrpE, a PPP protein phosphatase from Bacillus subtilis with unusual substrate specificity. Biochem J 366:929-936

Jijang M, Shao W, Perego M, Hoch JA (2000) Multiple histidine kinases regulate entry into stationary phase and sporulation in Bacillus subtilis. Mol Microbiol 38:535-542

Kaan T, Homuth G, Mäder U, Bandow J, Schweder T (2002) Genome-wide transcriptional profiling of the Bacillus subtilis cold-shock response. Microbiology 148:3441-3455

Kearns DB, Chu F, Branda SS, Kolter R, Losick R (2005) A master regulator for biofilm formation by Bacillus subtilis. Mol Microbiol 55:739-749

Keijser BJ, Ter Beek A, Rauwerda H, Schuren F, Montijn R, van der Spek H, Brul S (2007) Analysis of temporal gene expression during Bacillus subtilis spore germination and outgrowth. J Bacteriol 189:3624-3634

Levin PA, Losick R (1996) Transcription factor Spo0A switches the localization of the cell division protein FtsZ from a medial to a bipolar pattern in Bacillus subtilis. Genes Dev 10:476-488

Londoño-Vallejo JA, Fréhel C, Stragier P (1997) SpoIIQ, a foresporeexpressed gene required for engulfment in Bacillus subtilis. Mol Microbiol 24:29-39

Lopez D, Vlamakis V, Kolter R (2008) Generation of multiple cell types in Bacillus subtilis. FEMS Microbiol Rev 32:152-163

Mäder U, Schmeisky AG, Flórez LA, Stülke J (2012) SubtiWiki-a comprehensive community resource for the model organism Bacillus subtilis. Nucleic Acids Res 40(Database issue):D12781287. doi:10.1093/nar/gkr923

Márquez LM, Helmann JD, Ferrari E, Parker HM, Ordal GW, Chamberlin MJ (1990) Studies of sigma D-dependent functions in Bacillus subtilis. J Bacteriol 172:3435-3443

Mirel DB, Chamberlin MJ (1989) The Bacillus subtilis flagellin gene ( $h a g$ ) is transcribed by the sigma 28 form of RNA polymerase. J Bacteriol 171:3095-3101

Mirouze N, Desai Y, Raj A, Dubnau D (2012) Spo0A P imposes a temporal gate for the bimodal expression of competence in Bacillus subtilis. PLoS Genet 8:e1002586

Moir A, Kemp EH, Robinson C, Corfe BM (1994) The genetic analysis of bacterial spore germination. Soc Appl Bacteriol Symp Ser 23:9S-16S

Moir A, Corfe BM, Behravan J (2002) Spore germination. Cell Mol Life Sci 59:403-409

Molle V, Fujita M, Jensten ST, Eichenberger P, Gonzales-Pastor JE, Liu JS, Losick R (2003) The Spo0A regulon of Bacillus subtilis. Mol Microbiol 50:1425-1432

Nicholson WL, Munakata N, Horneck G, Melosh HJ, Setlow P (2000) Resistance of Bacillus subtilis endospores to extreme terrestrial and extraterrestrial environments. Microbiol Mol Biol Rev 46:548-572

Nishihara T, Freese E (1975) Motility of Bacillus subtilis during growth and sporulation. J Bacteriol 123:366-371

Ohashi Y, Inaoka T, Kasai K, Ito Y, Okamoto S, Satsu H, Tozawa Y, Kawamura F, Ochi K (2003) Expression profiling of translationassociated genes in sporulating Bacillus subtilis and consequence of sporulation by gene inactivation. Biosci Biotechnol Biochem 67:2245-2253

Ohlsen KL, Grimsley JK, Hoch JA (1994) Deactivation of the sporulation transcription factor SpoOA by the SpoOE protein phosphatase. Proc Natl Acad Sci USA 91:1756-1760

Paidhungat M, Setlow P (2000) Role of Ger proteins in nutrient and nonnutrient triggering of spore germination in Bacillus subtilis. J Bacteriol 182:2513-2519

Parashar V, Mirouze N, Dubnau DA, Neiditch MB (2011) Structural basis of response regulator dephosphorylation by Rap phosphatases. PLoS Biol 9:e1000589

Perego M (2001) A new family of aspartyl phosphate phosphatases targeting the sporulation transcription factor Spo0A of Bacillus subtilis. Mol Microbiol 42:133-143

Perego M, Brannigan JA (2001) Pentapeptide regulation of aspartylphosphate phosphatases. Peptides 22:1541-1547

Perego M, Hoch JA (1991) Negative regulation of Bacillus subtilis sporulation by the spoOE gene product. J Bacteriol 173:2514-2520

Pottathil M, Lazazzera BA (2003) The extracellular Phr peptide-Rap phosphatase signaling circuit of Bacillus subtilis. Front Biosci 8:d32-d45

R Development Core Team (2011) R: A language and environment for statistical computing. R Foundation for Statistical Computing, Vienna, Austria. ISBN 3-900051-07-0. http://www.Rproject.org/. Accessed 22 Feb 2013 
Saeed AI, Sharov V, White J, Li J, Liang W, Bhagabati N, Braisted J, Klapa M, Currier T, Thiagarajan M, Sturn A, Snuffin M, Rezantsev A, Popov D, Ryltsov A, Kostukovich E, Borisovsky I, Liu Z, Vinsavich A, Trush V, Quackenbush J (2003) TM4: a free, open-source system for microarray data management and analysis. Biotechniques 34:374-378

Schmalisch M, Maiques E, Nikolov L, Camp AH, Chevreux B, Muffler A, Rodriguez S, Perkins J, Losick R (2010) Small genes under sporulation control in the Bacillus subtilis genome. J Bacteriol 192:5402-5412

Setlow B, Cowan AE, Setlow P (2003) Germination of spores of Bacillus subtilis with dodecylamine. J Appl Microbiol 95: 637-648

Shah IM, Laaberki MH, Popham DL, Dworkin J (2008) A eukaryoticlike Ser/Thr kinase signals bacteria to exit dormancy in response to peptidoglycan fragments. Cell 135:486-496

Smits WK, Kuipers OP, Veening JW (2006) Phenotypic variation in bacteria: the role of feedback regulation. Nat Rev Microbiol 4:259-271

Steil L, Serrano M, Henriques AO, Völker U (2005) Genome-wide analysis of temporally regulated and compartment-specific gene expression in sporulating cells of Bacillus subtilis. Microbiology 151:399-420

Stragier P (2006) To kill but not be killed: a delicate balance. Cell 124:461-463

Stragier P, Losick R (1996) Molecular genetics of sporulation in Bacillus subtilis. Annu Rev Genet 30:297-341
Takamatsu H, Watabe K (2002) Assembly and genetics of spore protective structures. Cell Mol Life Sci 59:434-444

Tsukahara K, Ogura M (2008) Promoter selectivity of the Bacillus subtilis response regulator $\mathrm{DegU}$, a positive regulator of the fla/ che operon and sacB. BMC Microbiol 8:8

Tusher VG, Tibshirani R, Chu G (2001) Significance analysis of microarrays applied to the ionizing radiation response. Proc Natl Acad Sci USA 98:5116-5121

van Sinderen D, Venema G (1994) $\operatorname{comK}$ acts as an autoregulatory control switch in the signal transduction route to competence in Bacillus subtilis. J Bacteriol 176:5762-5770

van Sinderen D, Luttinger A, Kong L, Dubnau D, Venema G, Hamoen L (1995) comK encodes the competence transcription factor, the key regulatory protein for competence development in Bacillus subtilis. Mol Microbiol 15:455-462

Veening JW, Stewart EJ, Berngruber TW, Taddei F, Kuipers OP, Hamoen LW (2008) Bet-hedging and epigenetic inheritance in bacterial cell development. Proc Natl Acad Sci USA 105: 4393-4398

Zhang JQ, Griffiths KK, Cowan A, Setlow P, Yu J (2013) Expression level of Bacillus subtilis germinant receptor determines the average rate but not the heterogeneity of spore germination. J Bacteriol. doi:10.1128/JB.02212-12

Zuberi RA, Moir A, Feavers IM (1987) The nucleotide sequence and gene organization of the gerA spore germination operon of Bacillus subtilis 168. Gene 51:1-11 